\title{
Brain Tumor Segmentation using FCM and Symbolic Feature
}

\author{
Manjunath S, Sanja Pande M B, Raveesh B N
}

\begin{abstract}
The brain tumor segmentation from image is interesting and challenging in the field of image processing and pattern recognition. An early detection of a brain tumor region helps the patient to take the correct medicine and increase the rate of the survival.The brain tumor segmentation is a process of differentiating the abnormal tissues and normal tissues. most common types of brain tumors are Benign and Malignant tumors. In this paper, the Fuzzy C-Means (FCM) approach is used to cluster the abnormal cells region and normal cells region in the brain image. The possible noises are removed by employing the median filter and morphological function is applied to extract the possible tumor region. The true tumor region is extracted with the help of symbolic features. Finally, the proposed methods is tested on T2weighted MR brain images.
\end{abstract}

Keywords. Tumor, Fuzzy C Means, Symbolic, Segmentation.

\section{INTRODUCTION}

For the purpose of selective visualization and further analysis, Medical Image Processing deals with the development of problem-specific approaches to the enhancement of raw medical image data. Recently, medical image has undergone drastic positive changes due to the development of technologies. Nowadays, this improved technology helps to get the information about the human anatomy for many useful clinical applications. The brain tumor segmentation is a part of medical image processing. Due to the abnormal cells form within the brain, a br ain tumor or intracranial neoplasm occurs. The two ma in types of tumors are, Malignant and Benign tumors. The size and the position of the tumor within the brai $\mathrm{n}$ can be visualized by segmentation and volume meas urement of brain tumors. It also helps to compare bet ween the pre-operative and post- operative images or visualization of changes during treatment time period. Now, medical imaging technique play a vital role in $\mathrm{c}$ ancer diagnosis.

Revised Manuscript Received on July 22, 2019.

ManjunathS, Research Scholar, Department of Computer Science, Jain University, Bangalore, Karnataka, India

Dr SanjaPande M B, Professor and Head ,Department of Computer Science, GMIT, Davangere, Karnataka, India

Dr Raveesh B N, Professor and Head ,Department of Psychiatry, Mysore Medical College, Mysuru, Karnataka, India
One of the most used techniques to identify and locate the tumor in the brain is Magnetic Resonance Imaging (MRI). By applying image processing techniques the images obtained by medical imaging technique can bec ome a better quality image. MRI Brain image segmentation consist of extracting grey matter (GM), white matter (WM) and cerebrospinal fluid (CSF). It is always possible to detect the infected tissues easil $\mathrm{y}$ in the current medical field. However, the accuracy and the characteristics of abnormalities of the tumor tis sues are not precise. In the past, many researchers hav e proposed semiautomatic and fully automatic methods of brain tumor segmentation in the field of medical im aging. The amount of precision about the detection of tumor tissues leads to acceptance of a particular image segmentation method. The detection and segmentation of brain tumors are of great significance, and also ther $\mathrm{e}$ are problems and risks in the process.MRI is mainly used for brain tumor diagnosis and treatment in the $\mathrm{c}$ linic. MRI

offers various beneficial features like multiplanar capabi lities, potential of tissue characterization and no bone a nd teeth artifacts.

\section{RELATED WORKS}

Shen et. al., [1] presented a concurrent Fully Convolutional Networks for the brain tumor segmentation. This study propound a concurrent Fully Convolutional Networks(CFCN).

Gaussian filter, Mean filter and Median filter are chose $\mathrm{n}$ to pre-process the original multimodal MR

images. Then, the results from three networks are fuse d.Ultimately, a Fully Connected Conditional Random Fi eld (Fully Connected CRF)

is used to attain the postprocessing, improving the mod el's ability of detecting minute structures. The model was upskilled and assessed on BRATS

2015 challenge dataset. Havaei et. al., [2] introduced a fully automatic brain tumor segmentation method based on Deep Neural Networks(DNNs).

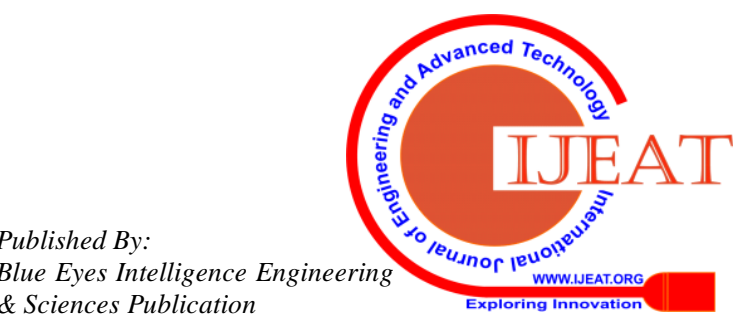




\section{Brain Tumor Segmentation using FCM and Symbolic Feature}

DNNs exhibits a novel CNN architecture which differs from those traditionally used in computer vision. At th e same time, The CNN utilizes both local features as well as more global contextual features. And also,the $n$ etworks use a final layer which is a convolution imple mentation of a fully connected layer that allows a 40 fold speed up which is different from most traditional uses of CNNs. aThe 2-phase

training procedures are discussed to tackle difficulties $r$ elated to the imbalance of tumor labels. Pareira et. al., [3]

proposed that brain tumors are life threatening and im mediate treatment is inevitable. The use of small kerne ls allows designing a deeper architecture, besides havin $\mathrm{g}$ a positive effect against over fitting, given the fewer number of weights in the network. This proposal was validated in the Brain Tumor Segmentation Challenge database BRATS 2013 and BRATS 2015. AbdelMaksoud et. al., [4]

propounded that development of techniques of medical image segmentation is necessary. This study presents a $\mathrm{n}$ efficient image segmentation approach using $\mathrm{K}$ means clustering technique integrated with Fuzzy Cmeans algorithm. Further,it is followed by thresholding and level set segmentation stages to provide an apt $b$ rain tumor detection. Liu et. al., [5]

discuss the brain tumor segmentation methods to separa te the different tumor tissues such as active cells, necr otic core, and edema from normal brain tissues of Wh ite Matter (WM),

and Cerebrospinal Fluid (CSF). Gordillo et. al., [6] presented the importance os semiautomatic and fully au tomatic methods. This research presents an overview of the most relevant brain tumor segmentation methods, conducted after the acquisition of the image. Given the advantages of magnetic resonance imaging over other diagnostic imaging, this survey is focused on MRI brain tumor segmentation. Selvakumar et. al., [7] emphasized that the study deals with the implementatio $\mathrm{n}$ of simple algorithm for detection of range and shape of tumor in brain MR images. Zhang et, al., [8] developed an algorithm for detecting the accurate size and shape of the brain tumor. The Support Vector Ma chine (SVM)

classification integrated with a selection of the features in a kernel space is proposed. The selection criteria a re defined by the kernel class separability. Based on $t$ his SVM

classification a framework to follow up the brain tumo $r$ evolution is proposed, which consists of the followin g steps: (1)

to learn the brain tumor and select the features from $t$ he first MRI examination of the patients; (2) to automatically segment the tumor in new data using SVM; to refine the tumor contour by a region growing techn ique. The system has been tested on real patient image $\mathrm{s}$ with satisfying results. The quantitative evaluations $b$ y comparing with experts manual traces and with other approaches demonstrate the effectiveness of the propos ed method. Menze et, al., [9]

presented that the study introduces a generative probabi listic model for segmentation of tumors in multi-

dimensional images. The model allows for different tu mor boundaries in each channel, reflecting difference $i$ $\mathrm{n}$ tumor appearance across modalities. It extends a pro babilistic atlas of healthy tissue priors with a latent atl as of the lesion and it also derives the estimation algo rithm to extract tumor boundaries and the latent atlas $\mathrm{f}$ rom the image data. It presents experiments on 25 glioma patient data sets, demonstrating significant impr ovement over the traditional multivariate tumor segment action.

\section{PROPOSED METHODOLOGY}

In this paper, the Fuzzy C-Means (FCM) algorithm is employed to produce the clustering region of the tumor in the brain image. The median filter and morphological function is applied to extract the possible tumor region. Symbolic features are used to isolate the true tumor region from the brain image. Finally, the proposed methods is tested on T2-weighted MR brain images.

\subsection{Fuzzy C-Means Algorithm}

The Fuzzy C-Means clustering method is employed to cluster. The cluster method divides the data points in to similar type of classes, hence inter class similarity will be low and intra class similarity will be high. In common clustering method, each data element will be a member of exactly one class but in fuzzy clustering, each data elements associated with membership levels and that data element may be member of more than one class. The Fuzzy C-Means is popular fuzzy clustering algorithm to make the partition of $\mathrm{n}$ elements into a collection of c fuzzy clusters based on some criterion. In this article, the FCM is employed to classify the input data into the tumor cluster with the lowest membership value. The fuzzy c-means algorithm assigns the membership levels for each data point corresponds to each cluster center based on the data point and the distance between the cluster centers. The summation of membership of each data point is equal to one. The fuzzy c-means algorithm allows one data element may be a member of two or more classes. The FCM gives best result for overlapped data elements and it is better than k-means algorithm. Figure 1 shows the procedure of possible tumor region, figure 1(a) is the input image, figure 1(b) represents the result of the FCM and figure 1(c) is the out come of median filter. Where equation 1 calculates the fuzzy membership $\mu \mathrm{ij}$, equation 2 computes the fuzzy center $\mathrm{Vj}$ and equation 3 is minimization function. 


$$
\mu_{i j}=\frac{1}{\sum_{k=1}^{c}\left(\frac{d_{i j}}{d_{i k}}\right)^{2 / m-1}}
$$

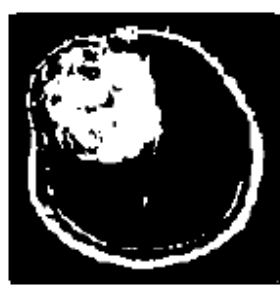$$
V_{j}=\frac{\sum_{i=1}^{n}\left(\mu_{i j}\right)^{m} x_{i}}{\sum_{i=1}^{n}\left(\mu_{i j}\right)^{m}}, \forall j=1,2,3 \ldots c
$$

$$
J(U, V)=\sum_{i=1}^{n} \sum_{j=1}^{c}\left(\mu_{i j}\right)^{m}\left\|x_{i}-V_{j}\right\|^{2}
$$

Where: ' $n$ ' is the number of data points.

'vj' represents the jth cluster center.

' $\mathrm{m}$ ' is the fuzziness index

'c' represents the number of cluster center.

'Â,tij' represents the membership of ith data to jth cluster center.

'dij' represents the Euclidean distance between ith data and $\mathrm{j}$ th cluster center.

' $\|\mathrm{xi}-\mathrm{Vj}\|$ ' is the Euclidean distance between ith data and $\mathrm{j}$ th cluster center.

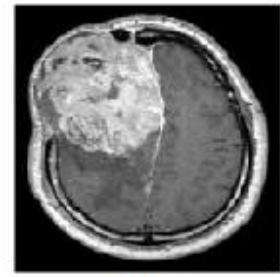

(a)

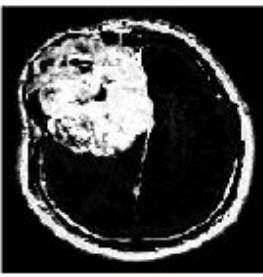

(b)

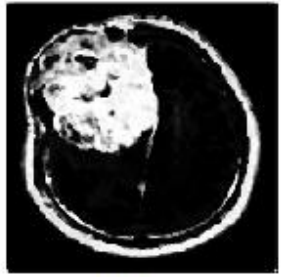

(c)
Figure 1. Extraction of possible tumor components

\subsection{Extraction of Possible Tumor Region}

A non linear filter such as median filter is applied to eliminate the noises by retaining tumor region. The median filter replaces the pixel value by calculating the median of its neighbor pixels, so that the brain tumor region will be enhanced from the non tumor region. The binary level information is extracted from the resultant image of the median filter to highlights the brightness region and to suppress a darken region. This binary level information increases the gap between tumor region and non tumor region in the brain image. The morphological function is effectively used to eliminate the non affected tumor region in the brightness region. The outcome of morphological function is considered as possible tumor region. Figure 2 depicts the process of true tumor region extraction. Figure 2(a) shows the binary level information, figure 2(b) refers the elimination of small components and figure 3 is the final output of the proposed method. procedure. (a)

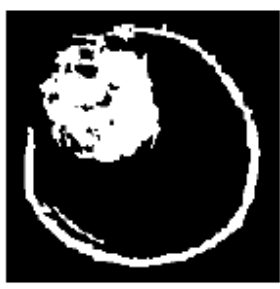

(b)

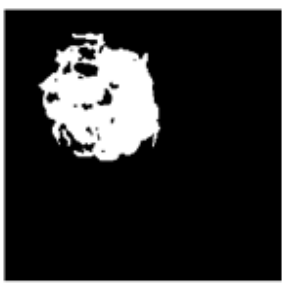

(c)
Figure 2. Extraction of true tumor components

\subsection{Segmentation of True Tumor Region}

True tumor region is extracted on the basis of multivalued symbolic feature representation. Usually, symbolic data is represented as crisp, multivalued, multivalued with weights, interval, continuous ratio and quantitative forms. The symbolic features effectively represents the shape information. More details and applications of symbolic data can be found in [10]. Hence, the symbolic data analysis concept has employed on medical image to extract the tumor region. The individual components of possible tumor regions are symbolized with the help of low level features like solidity and extent. The solidity (equation 4) of an image means the convexity of the image. The solidity of the object is equal to the area of a particular object divided by convex hull area of that object. Extent (equation 5) of an object is derived as area of a particular object divided by the area of its bounding rectangle. Hence, solidity and extent are the two main features to isolate the true tumor region from the possible tumor region.

$$
\text { Solidity }=\frac{\text { AreaofObject }}{\text { Convexhullareaofobject }}
$$

$$
\text { Extent }=\frac{\text { AreaofObject }}{\text { Areaofboundingrectangle }}
$$

\subsection{Classification of a Tumor}

The wavelet transform, pca and kernel SVM have been used to determine the type of tumor present in the brain. The wavelet transform results the highpass filtered information, which describes the local changes of a brightness in the tumor region. The wavelet transformation provides the approximation, horizontal, vertical and diagonal information. Texture and statistical features also extracted in addition to the symbolic features to improve the classification of a tumor type. Principal Component Analysis (PCA) is then employed to reduce the dimension of features. Kernel SVM is used to classify the type of tumor in the brain image. The basic SVM approach solves the two class classification problem of different types of data located at the different sides of a hyper plane. The kernel concept employed to the basic SVM. The kernel SVM approach is normally as same as basic SVM, except the dot product. The nonlinear kernel function replaces that dot product

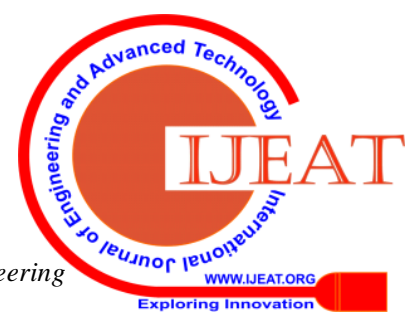




\section{Brain Tumor Segmentation using FCM and Symbolic Feature}

On the other hand, the Kernel SVM allows to the maximumboundary hyper plane in a decomposed feature space. The transformation is a nonlinear kernel function. Hence, the hyper plane drawn in the higher-dimensional feature space.

\section{EXPERIMENTAL RESULTS}

The proposed method is tested on T2-weighted MR brain images. This dataset consists two types of tumors i.e. Benign (non-cancerous) and Malignant (cancerous). The performance of the proposed method is computed using performing measures like, precision (equation 7), recall (equation 6) and f-measure (equation 8). The tumor segmentation parameters are labeled into three categories: (1) Actual Tumor Block (ATB) represents full region of the tumor present in an image, (2) Truly Detected tumor Block (TDB) is a tumor information detected by the proposed algorithm, and (3) Falsely Detected tumor Block (FDB) means non-tumor information detected by the proposed algorithm. The proposed method applied the fuzzy c-means algorithm and median filter to extract the prominent tumor components. Symbolized solidity and extent features help us to extract the true tumor candidates from the resultant of possible tumor candidates.

$$
\operatorname{Recall}(R)=\frac{T D B}{A T B}
$$

$$
\operatorname{Precision}(P)=\frac{T D B}{T D B+F D B}
$$

$F-\operatorname{measure}(F)=\frac{2 R P}{R+P}$

\subsection{Experimental Results on Benign Tumor}

A benign tumor is non-cancerous tumor. This type of tumor is not spread out throughout the body. This type of tumor develops when cells in the body divide and grow at an excessive rate. Each case of a benign tumor is individually reviewed to determine if treatment is necessary. Unlike malignant tumors, some benign tumors can be left alone and never cause any harm. Figure 3 shows the benign tumor results of the proposed method. The proposed method achieved recall is $92.3 \%$, precision is $94.5 \%$ and f-measure is $93.3 \%$.
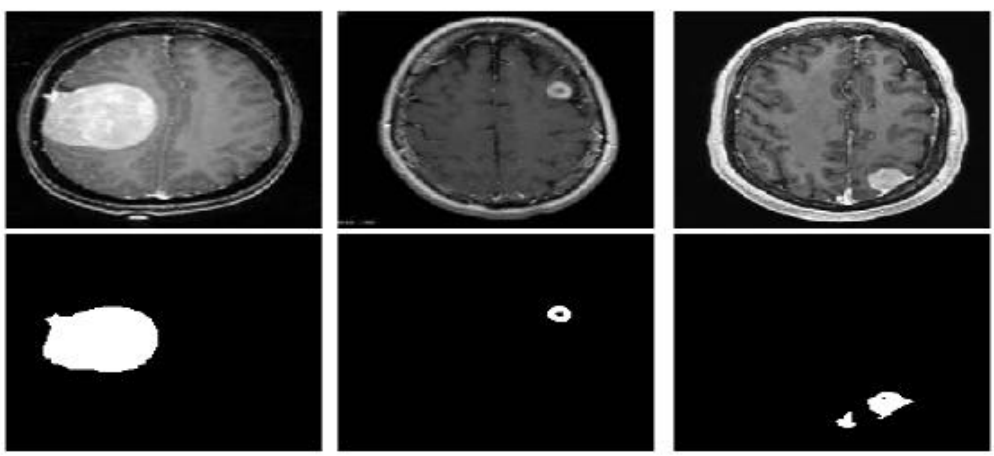

Figure 3. Inputs and corresponding outputs of Benign Tumor

\subsection{Experimental Results on Malignant Tumor}

Malignant tumor determined as cancerous tumor. Malignant is a Latin word for badly born. The malignant type of tumor multiplies uncontrollably and it spreads to various parts of the human body. This type of tumor formed by abnormal cells and it grows quickly. Malignant tumor is very dangerous as compare to the Benign Tumor. Figure 4 shows the malignant tumor results of the proposed method. The proposed method achieved recall is $92.1 \%$, precision is $89.5 \%$ and $\mathrm{f}-$ measure is $90.8 \%$.
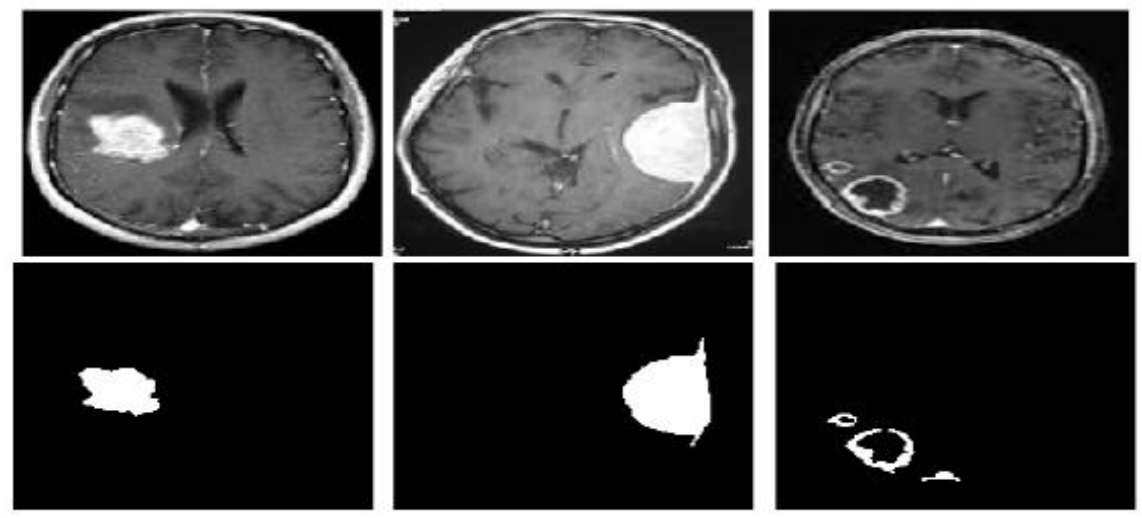

Figure 4. Inputs and corresponding outputs of Malignant Tumor

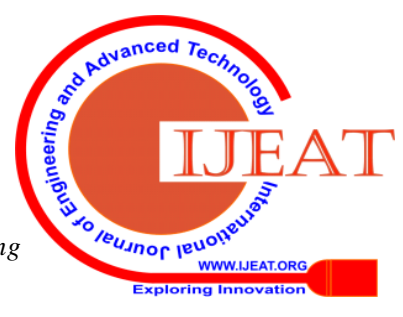




\section{CONCLUSION AND PERSPECTIVES}

The proposed method introduced a new symbolic analysis to extract the two types of tumor in brain image. Initially, the lowest membership value of fuzzy c-means algorithm effectively cluster the tumor affected region. The possible tumor region is identified by suppressing non tumor region with the help of median filter and morphological functions. Finally, true tumor region is determined by applying solidity and extent as a symbolic features. The proposed method is evaluated on T2-weighted MR brain images. In future work, the effective and efficient transformation method needs to be developed for distinguishing the tumor and non-tumor region.

\section{REFERENCES}

[1] Shen, G., Ding, Y., Lan, T., Chen, H., \& Qin, Z. (2018, March). Brain Tumor Segmentation Using Concurrent Fully Convolutional Networks and Conditional Random Fields. In Proceedings of the 3rd International Conference on Multimedia and Image Processing (pp. 24-30). ACM.

[2] Havaei, M., Davy, A., Warde-Farley, D., Biard, A., Courville, A., Bengio, Y., \&Larochelle, H. (2017). Brain tumor segmentation with deep neural networks. Medical image analysis, 35, 18-31.

[3] Pereira, S., Pinto, A., Alves, V., \& Silva, C. A. (2016). Brain tumor segmentation using convolutional neural networks in MRI images. IEEE transactions on medical imaging, 35(5), 12401251 .

[4] Abdel-Maksoud, E., Elmogy, M., \& Al-Awadi, R. (2015). Brain tumor segmentation based on a hybrid clustering technique. Egyptian Informatics Journal, 16(1), 71-81.

[5] Liu, J., Li, M., Wang, J., Wu, F., Liu, T., \& Pan, Y. (2014). A survey of MRI-based brain tumor segmentation methods. Tsinghua Science and Technology, 19(6), 578-595.

[6] Gordillo, N., Montseny, E., \&Sobrevilla, P. (2013). State of the art survey on MRI brain tumor segmentation. Magnetic resonance imaging, 31(8), 1426-1438.

[7] Selvakumar, J., Lakshmi, A., \&Arivoli, T. (2012, March). Brain tumor segmentation and its area calculation in brain MR images using K-mean clustering and Fuzzy C-mean algorithm. In Advances in Engineering, Science and Management (ICAESM), 2012 International Conference on (pp. 186-190). IEEE.

[8] Zhang, N., Ruan, S., Lebonvallet, S., Liao, Q., \& Zhu, Y. (2011). Kernel feature selection to fuse multispectral MRI images for brain tumor segmentation. Computer Vision and Image Understanding, 115(2), 256-269.

[9] Menze, B. H., Van Leemput, K., Lashkari, D., Weber, M. A., Ayache, N., \&Golland, P. (2010, September). A generative model for brain tumor segmentation in multi-modal images. In International Conference on Medical Image Computing and Computer-Assisted Intervention (pp. 151-159). Springer, Berlin, Heidelberg.

[10] Billard, L., \&Diday, E. (2006). Symbolic Data Analysis: Conceptual Statistics and Data Mining John Wiley 